\title{
Reformas laborales y estrategias sindicales. Claves de análisis a partir del caso del Sindicato dos Metalúgicos do ABC en Brasil
}

\author{
Labour reforms and union strategies. Analysis keys based on the case of the \\ Sindicato dos Metalúgicos do ABC in Brazil
}

\section{Lucila D’Urso \\ lucilafdurso@gmail.com \\ Universidad de Buenos Aires \\ Universidad Nacional del Centro de la Provincia de Buenos Aires, Argentina}

Recepción: 19 Diciembre 2019

Aprobación: 26 Junio 2020

Publicación: 01 Septiembre 2021

Cita sugerida: D’Urso, L. (2021). Reformas laborales y estrategias sindicales. Claves de análisis a partir del caso del Sindicato dos Metalúgicos do ABC en Brasil. Sociohistórica, 48, e143. https://doi.org/10.24215/18521606e143
Resumen: El presente artículo analiza la relación entre estrategias sindicales y reformas laborales. En tal dirección, se recupera la experiencia del Sindicato dos Metalúrgicos de $A B C$ en Brasil durante los gobiernos del Partido dos Trabalhadores y en los años posteriores a la sanción de la reforma laboral. Para ello, estudiaremos la dinámica de la conflictividad laboral entre 2003 y 2013. Luego, focalizaremos en el posicionamiento del sindicato ante la reforma laboral e identificaremos continuidades y rupturas en su estrategia respecto a los años precedentes. Al considerar en el análisis elementos que remiten a la orientación política e ideológica del accionar sindical, es posible concluir que las modificaciones en las normativas laborales no sólo expresan una ofensiva de los sectores dominantes sobre históricas conquistas de la clase trabajadora, sino que también pueden legitimar estrategias sindicales tendientes a convalidar la flexibilización de las relaciones del trabajo.

Palabras clave: Estrategias sindicales, Reformas Laborales, Brasil.

Abstract: This article analyses the relationship between union strategies and labour reforms. In this direction, the research retrieves the experience of the Sindicato dos Metalurgicos do $A B C$ in Brazil during the governments of the Partido dos Trabalhadores and in the years following to the approval of the labour reform. To this aim, we will study the dynamics of labour conflict between 2003 and 2013. Then, we will focus on the union stance on the labour reform, identifying continuities and ruptures in its strategy with respect to the previous years. When considering elements referred to the political and ideological orientation of the union action, it is possible to conclude that the modifications in labour regulations not only express an offensive, from the dominant sectors, against historical working class' conquests, but they can also legitimize union strategies likely to validate the flexibility of labour relations.

Keywords: Union strategies, Labour reforms, Brazil. 


\section{INTRODUCCIÓN}

En el último tiempo, en el ámbito académico de la Argentina, comenzaron a adquirir relevancia los debates en torno a la reforma laboral. Algunas preguntas de investigación indagaron acerca de las modificaciones en los instrumentos normativos que regulan la relación capital-trabajo, tales como las leyes, convenios y acuerdos colectivos (Marticorena, 2018; Asociación de Abogados Laboralistas, 2018). Otros trabajos analizaron el efecto disciplinador de la crisis económica en el mercado de trabajo y esbozaron la hipótesis de una suerte de reforma laboral "de hecho" signada por el deterioro de las condiciones de trabajo y los salarios en un contexto marcado por el aumento del desempleo y la informalidad. Asimismo, frente al boom de las economías de plataforma (Rappi, Glovo, Uber, etc.), diversas investigaciones se interesaron por analizar las consecuencias adversas del cambio tecnológico en los derechos laborales individuales y colectivos (Bensusán, 2017; Del Bono, 2018).

A nivel local, este tipo de reflexiones se suscitaron durante el gobierno de la alianza Cambiemos (2005-2019), en el marco de sucesivos intentos de modificar las legislaciones laborales. En el plano regional, adquirió especial relevancia la aprobación de la reforma laboral en Brasil en el año 2017 a partir de la sanción de la ley 13467/2017 que altera 201 puntos de la CLT (código laboral de Brasil) y la ley 13429/2017 que liberaliza la tercerización y amplía el contrato temporal.

De acuerdo con Krein (2018), ambas leyes tienden a la flexibilización y descentralización de las relaciones del trabajo en tanto amplían las posibilidades de utilización de modalidades de contratación por tiempo determinado, promueven la individualización de la negociación colectiva, limitan la injerencia de las instituciones laborales, permiten el avance de mecanismos de remuneración variable y debilitan las formas de organización y representación de los sindicatos. Así, la reforma laboral, constituye un sistema que amplía la libertad y el poder del capital, y que produce efectos directos sobre la economía, el mercado de trabajo, la protección social y la forma de organización de la sociedad brasileña (Krein y Gimenez, 2018).

En este marco, el presente artículo tiene por objetivo analizar la relación entre estrategias sindicales y reformas laborales a partir del estudio de caso del Sindicato dos Metalúrgicos do $A B C$ (SM ABC). En esta dirección, caracterizaremos la estrategia desarrollada por el SM ABC durante los gobiernos del Partido dos Trabalhadores (PT) (2011 a 2014 y 2015 a 2016) para, a posteriori, analizar el posicionamiento del sindicato frente a la reforma laboral sancionada en 2017 bajo el gobierno de Michel Temer (2016-2018). Proponemos abordar las estrategias sindicales atendiendo a su relación con las condiciones económicas, políticas e institucionales, pero también contemplando las tradiciones organizativas, las orientaciones político-ideológicas y las formas de construcción gremial que, entre otras cuestiones, se expresan en las modalidades de acción, el rol de los dirigentes y delegados sindicales, y los mecanismos de toma de decisiones.

Siguiendo esta perspectiva, los cambios acontecidos en la economía y la orientación política del gobierno resultan claves para comprender las estrategias sindicales, en la medida en que permiten conocer el marco en el cual actúan los sindicatos y sus condicionantes estructurales. Sin embargo, dichos elementos no explican por sí mismos las variaciones existentes en la acción sindical (Upchurch y Mathers, 2012). Tal como evidencian diversas experiencias internacionales, la pérdida de derechos laborales y la desregulación de las relaciones laborales pueden fortalecer distintos tipos de estrategias (Connolly y Darlington, 2012; Fishwick y Connolly, 2018).

De acuerdo con Hyman (2001), es posible distinguir tres tipos de estrategias sindicales en respuesta a la erosión de los marcos institucionales nacionales que regulan las relaciones laborales: estrategias clasistas, de mercado y de sociedad. Las estrategias clasistas están basadas en una perspectiva anticapitalista, en la adopción de medidas de acción directa y en la consecución de protestas socio-políticas que trascienden las luchas sindicales; la estrategia de mercado refiere al comúnmente denominado sindicalismo de negocios, basado en la negociación y en la búsqueda de consensos entre los trabajadores y empleadores con el objetivo de alcanzar 
ganancias mutuas; finalmente, la estrategia de sociedad remite al sindicalismo socialdemócrata europeo, en el cual las organizaciones sindicales promueven mejoras graduales de bienestar social a través del Estado.

En el caso de Brasil, efectivamente, los procesos socioeconómicos y políticos suscitados recientemente operan como condición de posibilidad para que el capital busque su valorización mediante el deterioro de las condiciones de empleo y salario de la clase trabajadora. En este cuadro, resulta clave indagar acerca de los alcances y límites del accionar sindical frente a los procesos de reforma laboral. Pero esto no implica analizar únicamente las respuestas que los sindicatos configuran frente a la ofensiva capitalista actual, sino también recuperar la relación existente entre reformas laborales y estrategias sindicales. La pregunta que surge, entonces, es cómo abordar esta relación sin ubicar al movimiento sindical como un actor que meramente reacciona frente a presiones y/o condiciones externas sino como un sujeto que constituye las relaciones laborales y que incide en la configuración de sus normativas.

A fin de conocer la relevancia del SM ABC en la dinámica sindical y política de Brasil, en la primera sección del artículo caracterizaremos su estrategia en perspectiva histórica a partir de una revisión exhaustiva de fuentes secundarias y de información proveniente de entrevistas semiestructuradas a dirigentes y delegados sindicales. Luego, estudiaremos el accionar del sindicato entre los años 2003 y 2013 tomando como referencia el análisis de la dinámica huelguística del sector automotor en la Región Grande ABC a partir de información proveniente del Departamento Intersindical de Estatística e Estudos Socioeconômicos (DIEESE), cuyo abordaje fue complementado con la consulta de periódicos y publicaciones sindicales. Finalmente, analizaremos el posicionamiento del SM ABC frente a la reforma laboral e identificaremos los puntos de continuidad y aspectos novedosos que presenta su estrategia respecto a los años precedentes, considerando fundamentalmente información proveniente de medios de prensa y documentos sindicales.

$\mathrm{Al}$ analizar las estrategias sindicales en retrospectiva, es decir, considerando las características históricas de su configuración, es posible observar que las modificaciones en las normativas laborales no sólo expresan una ofensiva de los sectores dominantes sobre históricas conquistas de la clase trabajadora, sino que también pueden legitimar estrategias sindicales tendientes a convalidar la flexibilización de las relaciones del trabajo.

\section{Los trabajadores METALÚRgicos DEL ABC: DEL PROYECTO CLASISTA AL SINDICALISMO PROPOSITIVO}

\subsection{El surgimiento del novo sindicalismo}

Creado en el año 1933, el SM ABC representa a los trabajadores de los municipios de São Bernardo do Campo, Diadema, Ribeirão Pires y Rio Grande da Serra que, junto con las ciudades de Santo André, São Caetano do Sul y Mauá, integran la denominada Región Grande ABC, caracterizada por ser la principal zona industrial de Brasil. La ciudad de São Bernardo do Campo presenta una relevancia particular en nuestro caso de estudio debido a que allí se sitúan la sede principal del SM ABC y también de la Confederação Nacional dos Metalúrgicos (CNM/CUT). Asimismo, como desarrollaremos a continuación, São Bernardo do Campo fue el epicentro de uno de los procesos de organización sindical y política más importantes de Brasil.

Los trabajadores metalúrgicos de la Región Grande $\mathrm{ABC}$ y el SM ABC desempeñaron un rol central en la historia del movimiento obrero de Brasil. Las huelgas protagonizadas hacia fines de los años setenta y principios de los ochenta por los trabajadores metalúrgicos de São Bernardo do Campo dieron lugar al surgimiento de un movimiento radical, orientado a la democratización del país y a la conquista de mejores condiciones salariales y de trabajo, fenómeno que años más tarde se denominó novo sindicalismo (Sader, 1988). Las movilizaciones que se extendieron en distintos puntos del país daban cuenta de la emergencia de un proyecto político transformador que, finalmente, cristalizó en la conformación del PT en el año 1980 y de la Central Única dos Trabalhadores (CUT) en 1983. 
Con posterioridad a las jornadas de lucha de 1979 y 1980, el SM ABC continuó siendo un actor central en la dinámica de las relaciones laborales e, incluso, en la vida política de Brasil. Sin embargo, desde la recuperación de la democracia en el año 1985, es posible observar un cambio en la orientación de las acciones del sindicato. En este sentido, la estrategia clasista que signó el accionar del SM ABC durante los años de la dictadura perdió peso a lo largo de los años noventa frente a la configuración de una estrategia marcada por el predominio de la acción en el plano institucional y por el otorgamiento de concesiones al gobierno y al capital.

Ahora bien, ¿qué elementos caracterizaron la estrategia del SM ABC a lo largo del tiempo? ¿De qué modo podemos explicar los cambios que atravesó la estrategia del sindicato desde los años ochenta al período actual? El elemento distintivo del movimiento encabezado por los trabajadores metalúrgicos en los años ochenta fue el carácter político que asumieron las huelgas, característica que tiende a asociarse a diversos factores.

Por un lado, algunas perspectivas destacan el modo en que las huelgas desarrolladas principalmente en el estado de San Pablo se enfrentaron a la dictadura militar; es decir, sitúan la dimensión política en la lucha del movimiento obrero por la recuperación de la democracia (Noronha, 2009; Véras de Oliveira, 2011). Por otro lado, una serie de investigaciones destacan que el elemento singular de las huelgas protagonizadas por los metalúrgicos del $\mathrm{ABC}$ en los años ochenta fue la emergencia y desarrollo de un movimiento sindical que tuvo como expresión programática la defensa de la independencia de clase para la conquista de derechos. En este sentido, no sólo adquiere centralidad la lucha por la recuperación de la democracia sino también el carácter que asumieron las reivindicaciones de los trabajadores, en tanto expresaron una disputa contra la sobreexplotación del trabajo que puso de manifiesto el antagonismo de clase (Boito, 1994).

Las huelgas en la Región Grande $\mathrm{ABC}$ se desarrollaron bajo el régimen militar y en un contexto signado por el agotamiento de un modelo de crecimiento basado en el desarrollo industrial y en la internacionalización de los mercados. Tal modelo estaba marcado por el incremento de los despidos, la contracción de los salarios, la intensificación de los ritmos de trabajo y la represión dentro y fuera de las fábricas (Antunes, 1988).

El lugar desempañado por los trabajadores metalúrgicos del $\mathrm{ABC}$ y sus dirigentes resultó central no sólo en el curso de las jornadas de lucha de los años ochenta sino también en la posterior conformación de la CUT y del PT, organizaciones que se gestaron sobre la base de las mismas fuerzas políticas ligadas al novo sindicalismo. Así, la estrategia sindical del SM ABC en cierta forma se fusiona con la trayectoria de la central obrera. La CUT se crea en el año 1983, durante la realización del $1^{\circ}$ Congresso Nacional da Classe Trabalhadora (CONCLAT). Desde su surgimiento, el SM ABC desempeña un lugar central en el interior de la CUT, que se expresa tanto en términos de representatividad en la dirección de la central como en la conducción de su línea política (De Souza y Vieira Trópia, 2012).

\subsection{El camino hacia una estrategia sindical basada en la conciliación de intereses}

Si bien las bases políticas e ideológicas que dieron origen a la CUT se encontraban fuertemente arraigadas en el novo sindicalismo, con el tiempo fueron surgiendo distintos posicionamientos en el interior de la central. De acuerdo con Praun (2012), algunos sectores destacaban la importancia de mantener la estrategia clasista, signada por la consecución de medidas de acción directa y por la independencia del gobierno y los partidos políticos que había marcado el surgimiento de la CUT. No obstante, con el retorno de la democracia y el surgimiento de una serie de instancias tripartitas como las Cámaras Sectoriales, también cobró fuerza una estrategia que enfatizaba en la acción institucional y en la búsqueda de consensos entre empresarios y trabajadores. Este segundo posicionamiento, defendido por un sector mayoritario dentro del cual adquirieron relevancia los dirigentes metalúrgicos del $\mathrm{SM} A B C$, fue el que prevaleció en los años noventa (Véras de Oliveira, 2011).

La consolidación de un proyecto de orientación neoliberal y su proximidad a la central obrera Força Sindical (FS), surgida en elaño 1991, fueron dos elementos importantes que incidieron en el viraje de la estrategia de la CUT. Como señala Trópia (2009), el elemento distintivo de FS es promover una estrategia 
de resultados a través de un modelo de actuación sindical que descarta la huelga como instrumento de lucha y prioriza la negociación y la cooperación entre trabajadores y empresarios.

El cambio en la estrategia de los sindicatos cutistas, en especial del SM ABC, fue analizado por diversos investigadores y suscitó variadas interpretaciones. Ladosky, Ramalho y Rodrigues (2014) vinculan el desarrollo de una estrategia de menor enfrentamiento y más abierta a la negociación con el carácter de las medidas implementadas por los gobiernos de Fernando Collor de Mello (1990-1992) y Fernando Henrique Cardoso (1995-2002), signadas por la pérdida de derechos sociales, el aumento del desempleo, la flexibilización de las relaciones del trabajo y el ataque al movimiento sindical. Para otros autores, en cambio, lejos de adoptar un posicionamiento de simple adaptación a los cambios en materia laboral, los sindicatos cutistas comenzaron a participar activamente en la implementación de las reformas del programa neoliberal (Praun, 2012; Galvão, 1998 y 2012).

El ejemplo más expresivo de esto lo constituyen las Cámaras Sectoriales, instancias tripartitas implementadas en los años 1992 y 1993. Desde el punto de vista del sindicato, las Cámaras Sectoriales buscaron sustituir el proceso de reestructuración productiva llevado a cabo de forma unilateral por las empresas, a cambio de una reestructuración negociada que garantizaría el mantenimiento de los puestos de trabajo y una mejor distribución de las ganancias provenientes de la creciente productividad de aquellas (Praun, 2012). Sin embargo, como señala Galvão (2002), las Cámaras Sectoriales consolidaron una modalidad de sindicalismo propositivo, caracterizada por el predominio de la acción en el plano institucional y por el otorgamiento de concesiones al gobierno y al capital (Galvão, 2012, p. 135).

$\mathrm{Al}$ posicionamiento que asume $\mathrm{SM} A B C$ en las instancias de diálogo social promovidas por el gobierno y las empresas se adiciona un nuevo elemento: el sindicato comienza a desempeñar una función de prestador de servicios. En el año 1996, y bajo la órbita del sindicato, se crea la firma Unisol, con el objetivo de recuperar empresas en quiebra mediante cooperativas de producción. Asimismo, en el año 1997 el SM ABC participa junto a los gobiernos municipales, estatales y empresarios locales de la Cámara Regional de ABC, creada con el objetivo de estimular la inversión y el desarrollo en la región. La Cámara constituyó grupos de trabajo para diagnosticar problemas vinculados con temas como educación, salud, medio ambiente y cultura, y diseñar proyectos tendientes a su solución. En este marco, el sindicato propuso la creación del MOVA (Movimiento de Alfabetización Regional del $\mathrm{ABC}$ ). Finalmente, el ejemplo más expresivo lo constituye la creación de agencias de empleo que, entre otras cuestiones, se ubicaron como intermediarias entre los trabajadores y las empresas, y ofrecieron cursos de formación profesional a los trabajadores desempleados.

Para algunos autores, como Véras de Oliveira (2011), el nuevo rol desempeñado por los sindicatos cutistas, y en particular por el SM ABC, a partir de los años noventa forma parte de una estrategia de lucha orientada a la defensa del empleo y de los derechos, que el autor denomina sindicalismo ciudadano. Desde esta perspectiva, en un contexto signado por el retiro de derechos por parte del Estado, los sindicatos comenzaron a brindar servicios en pos de suplir las necesidades sociales de los trabajadores de su categoría.

A la par que algunas organizaciones sindicales comenzaron a reemplazar funciones que históricamente estaban asociadas al rol del Estado, se observa el abandono del discurso antipatronal y anticapitalista que había signado al novo sindicalismo. De acuerdo con Galvão (2012), al sustituir la noción de clase por una concepción peculiar de ciudadanía, el sindicalismo propositivo permitió la aproximación de la CUT a FS, al tiempo que estimuló la colaboración y la conciliación de clases. Así, paulatinamente, la CUT -y con ella el SM ABC-abandonó su herencia clasista en pos del alcance de metas comunes con el gobierno y la patronal, y las disputas por las condiciones y ritmos de trabajo e, incluso, por el control del proceso de trabajo quedaron soslayadas.

Para los dirigentes sindicales, una vez conquistada la democracia el sindicato debía orientar su estrategia a la construcción de espacios de diálogo y consenso:

El SM ABC tiene que evaluar... siempre fue un sindicato auténtico, de lucha, que confrontó cuando tenía que hacerlo, inclusive en la época de la dictadura. Ahí, a veces, decíamos que el sindicato era radical. Pero realmente lo era, imaginate que la 
dictadura colocaba coroneles y policías para ser jefes de las empresas, entonces la cosa era bien pesada. Y nosotros, en esa época no estábamos preparados para negociar. Estábamos preparados para confrontar. Tuvimos ese momento de enfrentamiento y en los años noventa, con la crisis internacional, el sindicato comenzó a percibir también que no daba para vivir en una isla. Y desde el comienzo fue eso, desde la creación de la CUT, de las confederaciones y federaciones, la idea de fortalecer Brasil. Porque si no todo el mundo que estaba viviendo aquí [se refiere a las empresas ubicadas en la región $\mathrm{ABC}$ ] iba a cerrar (...). Una cosa es divisar el futuro, nosotros tenemos que proteger nuestra región, porque no sirve de nada estar peleando, peleando y perder todo (...). Porque pensar que sólo con el sindicato vamos a resolver los problemas del mundo... ¡no da! Entonces, el sindicato tiene un papel limitado; por eso nosotros estamos siempre en negociación (...) nosotros tenemos que avanzar dentro de eso, pero no descartamos la huelga. Cuando no queda otra, es la huelga. (Presidente de la CNM/CUT, 1/10/2014)

\subsection{La institucionalización del consenso en los años dos mil}

Los años dos mil trajeron consigo un nuevo escenario signado por la llegada del PT a la presidencia y la recuperación económica a nivel nacional en un contexto de crecimiento en América Latina. En este marco, el SM ABC y otras organizaciones sindicales ligadas a la CUT profundizaron su actuación institucional, lo cual estuvo especialmente estimulado por el ascenso de dirigentes sindicales al poder ejecutivo (Colombi, 2020). El caso de Luiz Inácio "Lula" da Silva, quien había sido presidente del SM de ABC durante las huelgas de 1979 y 1980, es la máxima expresión de esa tendencia, pero también se destacan otros casos como el de Luiz Marinho, que ocupó el cargo de presidente del sindicato entre 1993 y 2005, y de la CUT entre 2003 y 2005, y fue nombrado luego Ministro de Trabajo en el año 2005 y prefeito (intendente) de São Bernardo do Campo entre los años 2009 y 2016.

El SM ABC apoyó las sucesivas candidaturas a la presidencia y a los gobiernos del PT. Con el triunfo electoral de Lula en el año 2003, el SM ABC consolidó su posición como pilar fundamental de la CUT y, por tanto, del PT (Colombi y D’Urso, 2018; Galvão, 2012). La proximidad política e ideológica de la central sindical al gobierno propició la eclosión de conflictos en su interior que condujeron a la salida de dirigentes y activistas sindicales, y a la creación de nuevas centrales: en el año 2004 se creó CUNLUTAS; en 2006, la Intersindical, que en 2008 se fraccionó en dos centrales (Intersindical - Instrumento de Luta e Organização da ClasseTrabalhadora e Intersindical CCT - Central da Classe Trabalhadora); finalmente, en 2007, surgió la Central dos Trabalhadores e Trabalhadoras do Brasil (CTB).

Ahora bien, ¿qué características presentó la estrategia del SM ABC bajo los gobiernos del PT?; ¿cuáles fueron las formas de organización y acción colectiva de los trabajadores?; ¿en qué medida circunscribir el accionar sindical al plano de la lucha económica limitó la capacidad de organización del sindicato?

\section{El SM ABC DURANTE LOS gobiernos DEL PT}

\subsection{Consideraciones sobre el abordaje de las huelgas}

Para reconstruir la dinámica de las huelgas del sector automotor en la Región Grande ABC entre los años 2003 y 2013 recurrimos a datos provenientes del Sistema de Acompanhamento de Greves (SAG) del DIEESE, que releva información proveniente de noticias publicadas en los principales diarios impresos o electrónicos nacionales y en publicaciones sindicales. La unidad de relevamiento son las huelgas, definidas por el organismo como "una interrupción temporal del trabajo, efectuada intencionalmente por un grupo de trabajadores con el objetivo de imponer una reivindicación, oponerse a una exigencia o expresar una queja”. Es decir, quedan excluidas las paralizaciones de iniciativa patronal (lockouts), y las formas de protesta que no implican suspensión de trabajo, como el trabajo a reglamento o el trabajo a desgano.

A partir de la base de huelgas del SAG-DIEESE identificamos las siguientes dimensiones de análisis para cada huelga: año y mes de la huelga; sujeto que impulsa la acción (comisión de fábrica o empresa, 
sindicato, federación o confederación, central sindical); agregación económica (empresa, categoría, intercategoría); tipo de establecimiento (autopartista, terminal automotriz, talleres mecánicos, concesionarias, toda la categoría); tipo de reclamo, distinguiendo entre salarial (demanda de mejoras salariales genéricas, demanda de mejoras salariales específicas, pagos adeudados, negociación o paritaria salarial) y no salarial (despido o renovación de contrato, regularización del contrato laboral, condiciones y medio ambiente laboral, trato discriminatorio o sanciones, reclamos por representación, negociación no salarial, demandas de seguridad, otras demandas).

En cuanto a la agregación territorial, se contemplaron las huelgas del sector automotor llevadas a cabo en la totalidad de los municipios que integran la Región Grande $\mathrm{ABC}$, lo cual nos permitió constatar la relevancia del SM ABC en la dinámica huelguística de la región en el período estudiado (Cuadro 1).

CUADRO 1

Sindicatos y huelgas en el sector automotriz de la Región Grande ABC (2003-2013)

\begin{tabular}{|c|c|c|c|}
\hline Organización sindical & $\begin{array}{c}\text { Central } \\
\text { sindical }\end{array}$ & Ciudades & Huelgas \\
\hline $\begin{array}{c}\text { Sindicato dos Metalúrgicos } \\
\text { do ABC (SM ABC) }\end{array}$ & CUT & $\begin{array}{c}\text { São Bernardo do Campo/Diadema/Riberao } \\
\text { Pires /Rio Grande da Serra }\end{array}$ & 76 \\
\hline $\begin{array}{c}\text { Sindicato dos Metalúrgicos de } \\
\text { Santo André e Mauá }\end{array}$ & $\begin{array}{c}\text { Força } \\
\text { Sindical }\end{array}$ & $\begin{array}{c}\text { Santo André/ Mahúa / } \\
\text { Osasco }\end{array}$ & 14 \\
\hline $\begin{array}{c}\text { Sindicato dos Metalúrgicos de } \\
\text { São Caetano do Sul }\end{array}$ & $\begin{array}{c}\text { Força } \\
\text { Sindical }\end{array}$ & São Caetano do Sul & 3 \\
\hline $\begin{array}{c}\text { Federação dos Sindicatos de } \\
\text { Metalúrgicos da CUT (FEM) }\end{array}$ & CUT & Región Grande ABC & 2 \\
\hline $\begin{array}{c}\text { Sindicato dos Metalúrgicos de } \\
\text { São José dos Campos e Região }\end{array}$ & $\begin{array}{c}\text { CSP } \\
\text { Conlutas }\end{array}$ & $\begin{array}{c}\text { São José dos Campos / } \\
\text { Sudeste São Caetano do Sul }\end{array}$ & 1 \\
\hline Força Sindical & São Caetano do Sul & 1 \\
\hline
\end{tabular}

Elaboración propia sobre la base de datos del SAG-DIEESE

En Brasil la estructura de representación sindical es por rama de actividad y a nivel regional. Cada estado cuenta con organizaciones sindicales afiliadas a distintas confederaciones sectoriales que representan a los trabajadores en diversas ciudades. En el caso del SM ABC, su sede principal está ubicada en São Bernardo do Campo. El sindicato también cuenta con sedes ubicadas en las otras ciudades de la región donde tiene representación (Diadema, Ribeirão Pires y Rio Grande da Serra). Una particularidad de la estructura de representación del $S M A B C$, que la distingue de otros sindicatos, es que cuenta con representantes sindicales en los lugares de trabajo que integran Comisiones de Fábrica (CF) y/o Comités Sindicales de Empresa (CSE).

\subsection{Las huelgas en el sector automotriz de la Región grande ABC}

Las huelgas metalúrgicas de la Región Grande ABC Paulista representan la mayor parte de las huelgas en el sector privado de Brasil entre los años 2003 y 2013. A modo de ejemplo, en el año 2004 se realizaron 54 huelgas en el ámbito privado industrial; sobre ese total, el 78 \% se localizó en el estado de San Pablo, que forma parte de la Región Grande ABC. El 74\% de las huelgas situadas en el estado de San Pablo fueron impulsadas por trabajadores metalúrgicos (DIEESE, 2005).

En cuanto al tipo de reclamo, las huelgas en el sector privado estuvieron motivadas principalmente por reivindicaciones salariales. Se destacan aquellas vinculadas con la Participação nos Lucros e Resultados (PLR); es decir, con la participación de los trabajadores en las ganancias y resultados de las empresas. Este reclamo también impulsó gran parte de las acciones colectivas en la rama metalúrgica.

El incremento del número de huelgas motivadas por reclamos de tipo salarial es un elemento distintivo de la actividad huelguística brasileña a partir del año 2003 y marca un contrapunto con la configuración 
que presentaron las huelgas en la segunda mitad de la década del noventa, cuando estuvieron orientadas a mantener derechos adquiridos o a evitar su incumplimiento (Boito y Marcelino, 2010).

Esta diferenciación en el tipo de reclamo conllevó que diversos analistas vincularan la dinámica de las huelgas durante los gobiernos del PT con el desarrollo de una estrategia sindical ofensiva. En este sentido, Noronha (2009) señala que durante el gobierno de Lula las huelgas se consolidaron como un elemento colectivo de presión y negociación. En una dirección similar, Boito y Marcelino (2010) argumentan que el incremento del número de huelgas y la prevalencia de reivindicaciones salariales expresaron una recuperación de la actividad sindical en Brasil. No obstante, en un trabajo previo, los autores argumentan que las luchas sindicales quedaron circunscriptas en un nivel reivindicativo y localizado, sin presentar aspectos novedosos en términos de las formas de organización y la orientación política de las acciones (Boito, Galvão y Marcelino, 2011). De acuerdo con Atzeni y Ghiliani (2008), analizar el carácter de las huelgas implica observar no sólo su variación cuantitativa sino también los tipos de reclamos que las impulsan y una serie de dimensiones que remiten al nivel de organización de los trabajadores: nivel de agregación de los conflictos, sujetos que impulsan las acciones y formas de lucha adoptadas.

Tomando en consideración las investigaciones referenciadas, a continuación analizaremos las huelgas del sector automotor en la Región Grande $\mathrm{ABC}$ con el objetivo de conocer el tipo de estrategia sindical que el SM ABC configura entre los años 2003-2013. Para ello, identificamos tres subperíodos cuya delimitación se estableció considerando la distribución anual de las huelgas, los niveles de producción y empleo sectoriales (Tabla 1), los tipos de reclamos (Tabla 2), las modalidades de acción, el rol de los dirigentes y delegados sindicales y los mecanismos de toma de decisiones.

\section{(1) Primer subperiodo (2003-2008): campañas salariales y huelgas por categoria}

El primer subperíodo se extiende entre los años 2003 y 2008 y se caracteriza por la realización de intensas medidas de lucha cuyas reivindicaciones fueron fundamentalmente salariales, aunque también adquirieron relevancia demandas vinculadas con las condiciones de trabajo, la intensificación de los ritmos de producción y la flexibilización de la jornada laboral (Tabla 2). Si bien en este primer período es posible observar importantes variaciones en el número de conflictos de cada año y en los niveles de producción y empleo (Tabla 1), las huelgas presentaron características comunes que expresaron la capacidad de organización y movilización de los trabajadores y sindicatos del sector.

Durante los primeros dos años, el número de huelgas se mantuvo relativamente parejo ( 7 huelgas en el 2003 y 9 en el 2004). La diferencia entre un año y otro radica en el tipo de demandas (Tabla 2): en el año 2003 se destacan los conflictos por despidos y por cambios en las condiciones de trabajo mientras que hacia el año 2004, conforme se observa un crecimiento en los niveles de producción y empleo sectorial (Tabla 1), adquieren relevancia las huelgas por reivindicaciones de índole salarial (Tabla 2).

En el año 2004 se realizaron tres huelgas en el marco de campañas salariales, dos de las cuales implicaron a toda la categoría de trabajadores. Los acuerdos salariales fueron alcanzados luego de sucesivas paralizaciones conducidas por los sindicatos del sector. En este punto, se distingue la conjunción de dos elementos clave que caracterizan la dinámica huelguística del primer subperíodo: por un lado, una movilización activa por parte de las bases organizadas principalmente a través de las CF y, por otro lado, el rol desempeñado por los dirigentes sindicales en las rondas de negociación. En este sentido, resulta ilustrativa la caracterización del presidente del SM ABC José López Feijóo sobre los acuerdos salariales del año 2004: "Este acuerdo sólo fue posible por causa de la organización y capacidad de lucha de los metalúrgicos de $\mathrm{ABC}$ (...). Es esa capacidad de lucha la que, a partir de ahora, va a presionar a los grupos patronales que aún no negociaron". El dirigente sindical ubica en la capacidad de organización y lucha del SM ABC el punto clave del acuerdo salarial no sólo en términos de conquista para los trabajadores alcanzados sino también como pauta en las negociaciones aún abiertas. 
En el año 2005 las huelgas alcanzaron el nivel más elevado de todo el período estudiado. Este incremento se debe, principalmente, a la proliferación de huelgas por PLR en el marco de un incremento de la producción de automóviles (Tabla 1). Estas acciones fueron mayoritariamente impulsadas por el SM ABC en las terminales automotrices. En este marco, adquirió relevancia una huelga en Mercedes Benz, donde los trabajadores iniciaron un proceso de lucha para exigir que la empresa mejorase su propuesta de PLR acorde al incremento de la producción respecto al año previo. Para ello, se efectuaron desfiles en la planta que tuvieron el objetivo de ir sumando nuevos trabajadores a medida que la movilización se extendía en cada uno de los grupos de trabajo. En este sentido, más allá del tipo de reclamo que motivó las huelgas (PLR), en las terminales automotrices de Brasil cobra relevancia la modalidad que asumió el conflicto, porque expresa un interés particular por parte del actor sindical en promover la participación del conjunto de trabajadores organizados en el lugar de trabajo. También reviste importancia la realización de asambleas, la discusión de las propuestas de la empresa en el lugar de trabajo y el rol desempeñado por los representantes de las CF: "Terminada la reunión haremos una asamblea. Lo ideal será votar una propuesta que atienda a nuestros intereses. Caso contrario, debatiremos las medidas de lucha a tomar"1 "Esta paralización es el comienzo. La lucha sólo terminará cuando la empresa llame a los representantes de los trabajadores y presente una propuesta mejor" ${ }^{2}$.

En el año 2006 se desacelera el ritmo de crecimiento del sector respecto de los años previos. Asimismo, el empleo -que venía manteniendo una tendencia ascendente- también presenta una leve caída (Tabla 1). En este escenario, la mayor parte de las huelgas fueron por demandas salariales específicas (Tabla 2). Luego, en 2007, se incrementan la producción de automóviles y los niveles de empleo respecto del año precedente. En este escenario la conflictividad laboral desciende, pero vuelve a incrementarse en 2008 (Tabla 1), cuando los efectos de la crisis internacional comienzan a reflejarse en la producción y el empleo sectorial.

\section{(2) Segundo subperiodo (2009-2010): crisis y recuperación del sector}

En el año 2009 se llevaron a cabo 12 huelgas, de las cuales únicamente 3 fueron por reivindicaciones salariales (Tabla 2). Los conflictos laborales estuvieron signados por los vaivenes en la producción en el marco de la crisis económica internacional (Tabla 1). En la primera mitad del año 2009 se desarrollaron 5 huelgas situadas en empresas autopartistas, por causa de despidos fundamentados en una disminución del ritmo de crecimiento económico. Algunos de los casos más destacados se localizaron en las autopartistas TRW Automotive, Federal Mogul, Mahle e Isringhausen. El foco que desató los conflictos en estos establecimientos fue el anuncio de despidos sin previa negociación con el sindicato.

Pasado el impasse de la crisis económica internacional, el sector automotor comenzó a recuperar sus niveles de producción y empleo (Tabla 1). En este marco, en el año 2010 las luchas sindicales tuvieron el objetivo de recomponer los niveles salariales previos a la crisis: el SM ABC realizó 7 huelgas exigiendo incrementos salariales en las principales terminales automotrices de la región (Volkswagen, Mercedes Benz, Scania y Ford). En algunas de las autopartistas más importantes de la región (Mahle e Incodiesel), en cambio, las demandas por recomposiciones salariales estuvieron sujetas a la PLR.

\section{(3) Tercer subperiodo (2011-2013): la descentralización de la negociación colectiva y de las buelgas}

Las características que presentó la dinámica huelguística en el año 2010 cambiaron nuevamente en 2011 en el marco de un deterioro de la economía de Brasil y del sector automotriz en particular (Tabla 1). El número de huelgas descendió y los reclamos se vincularon con demandas salariales específicas o bien, con el despido de trabajadores en el marco de procesos de reorganización del proceso de trabajo. 
Asimismo, una singularidad del tercer subperíodo reside en que a partir del año 2011, en la rama metalúrgica, los acuerdos comenzaron a realizarse por empresa lo cual conllevó a un incremento de las huelgas situadas en el lugar de trabajo y en una reducción de aquellas acciones que implicaron a toda la categoría o rama de actividad (DIEESE, 2013). Esto marca una distinción con los primeros años del período, cuando las huelgas se caracterizaron por organizar a los trabajadores por categoría en intensos conflictos realizados en el marco de campañas salariales. A partir de 2011 en cambio, la mayor parte de las huelgas estuvieron motivadas por reclamos salariales vinculados con la PLR (Tabla 2).

De acuerdo con Krein y Teixeira (2014), los sindicatos percibieron que la PLR era la forma menos conflictiva de aumentar la remuneración de los trabajadores: la resistencia patronal resultaba menor, al tiempo que permitía legitimar al sindicato frente al conjunto de los trabajadores, debido al alcance de conquistas que efectivamente aumentaron el poder adquisitivo del salario de sus representados. Sin embargo, el principal problema de la PLR se ubica, justamente, en que está condicionada al alcance de objetivos y, por tanto, tiende a ejercer presión sobre las condiciones de trabajo al plantear exigencias en términos de conducta, calidad, producción, ventas, productividad, reducción de costos y rentabilidad (Krein, 2013).

TABLA 1

Variación interanual producción de automóviles, empleo y cantidad huelgas en el sector automotriz de la Región Grande ABC (2003-2013)

\begin{tabular}{|c|c|c|c|}
\hline Año & Producción de automóviles (\%) & $\begin{array}{c}\text { Empleo,registrado, } \\
(\%)\end{array}$ & $\begin{array}{c}\text { phitualgas feb } \\
\left(n^{\circ}\right)\end{array}$ \\
\hline 2003 & - & - & 7 \\
\hline 2004 & 24,5 & 12,3 & 9 \\
\hline 2005 & 11,4 & 6,1 & 13 \\
\hline 2006 & 2,4 & $-1,1$ & 10 \\
\hline 2007 & 16,4 & 11,9 & 3 \\
\hline 2008 & 5,8 & 5,3 & 9 \\
\hline 2009 & 2,8 & $-0,7$ & 12 \\
\hline 2010 & 4,5 & 7,9 & 7 \\
\hline 2011 & $-1,9$ & 5,9 & 4 \\
\hline 2012 & 5,0 & 6,0 & 12 \\
\hline 2013 & 6,9 & 2,5 & 11 \\
\hline
\end{tabular}


TABLA 2

Las huelgas del sector automotriz en la Región Grande

ABC según tipo de reclamo principal (2003-2013)

\begin{tabular}{|l|c|c|c|c|c|c|c|c|c|c|c|c|c|}
\hline & $\mathbf{2 0 0}$ & $\mathbf{2 0 0}$ & $\mathbf{2 0 0}$ & $\mathbf{2 0 0}$ & $\mathbf{2 0 0}$ & $\mathbf{2 0 0}$ & $\mathbf{2 0 0}$ & $\mathbf{2 0 1}$ & $\mathbf{2 0 1}$ & $\mathbf{2 0 1}$ & $\mathbf{2 0 1}$ & $\begin{array}{c}\text { Tot } \\
\text { al }\end{array}$ & $\begin{array}{c}\text { Tot } \\
\text { al } \\
(\%)\end{array}$ \\
\hline $\begin{array}{l}\text { Demandas } \\
\text { de } \\
\text { mejoras } \\
\text { salariales }\end{array}$ & 1 & 5 & 7 & 5 & 2 & 3 & 3 & 7 & 2 & 10 & 10 & $\mathbf{5 5}$ & 56,7 \\
\hline $\begin{array}{l}\text { Pagos } \\
\text { Adeudados }\end{array}$ & - & - & - & 1 & 1 & 1 & - & - & - & - & - & $\mathbf{3}$ & 3,1 \\
\hline $\begin{array}{l}\text { Despidos o } \\
\text { renovación } \\
\text { de contrato }\end{array}$ & 3 & - & 1 & 1 & - & 1 & 7 & - & 2 & 1 & 1 & $\mathbf{1 7}$ & 17,5 \\
\hline $\begin{array}{l}\text { Regularizaci } \\
\text { ón del } \\
\text { Contrato } \\
\text { Laboral }\end{array}$ & 1 & 1 & 2 & - & - & - & - & - & - & - & - & $\mathbf{4}$ & 4,1 \\
\hline $\begin{array}{l}\text { Condiciones } \\
\text { y Medio } \\
\text { Ambiente } \\
\text { Laboral }\end{array}$ & 1 & 2 & 1 & 3 & - & - & - & - & - & - & - & $\mathbf{7}$ & 7,2 \\
\hline $\begin{array}{l}\text { Trato } \\
\text { Discriminato } \\
\text { ro } \\
\text { O Sanciones }\end{array}$ & - & - & 2 & - & - & - & - & - & - & - & - & $\mathbf{2}$ & 2,1 \\
\hline $\begin{array}{l}\text { Demandas } \\
\text { de seguridad }\end{array}$ & 1 & - & - & - & - & 4 & 1 & - & - & - & - & $\mathbf{6}$ & 6,2 \\
\hline \begin{tabular}{l} 
Otros \\
\hline Total
\end{tabular} & - & 1 & - & - & - & - & 1 & - & - & 1 & - & $\mathbf{3}$ & 3,1 \\
\hline
\end{tabular}

Elaboración propia a partir de datos del SAG-DIEESE

El análisis presentado complejiza aquellas caracterizaciones que asociaron el incremento de las huelgas por reivindicaciones salariales a la configuración de una estrategia sindical ofensiva (Noronha, 2009; Boito y Marcelino, 2010). Al estudiar la dinámica huelguística del sector automotor de la Región Grande ABC, es posible observar que el accionar sindical no estuvo configurado únicamente en relación con los vaivenes en la producción y el empleo sectoriales. La incidencia de tales dimensiones estructurales en las características que presentaron las huelgas no resultó unidireccional, sino que presentó variaciones en cada subperíodo de acuerdo con la estrategia -de mayor o menor confrontación y movilización, y con un menor o mayor grado de descentralización de los conflictos- adoptada por el SM ABC.

\section{Los metalúrgicos Del ABC Frente a la REForma laboral EN Brasil}

En Brasil la reforma laboral entró en vigencia en noviembre del año 2017. Como señalan Colombi, Lemos y Krein (2018), la reforma gira en torno a dos ejes principales. En primer lugar, altera el lugar desempeñado por el Estado en la definición de las condiciones de contratación, uso y remuneración del trabajo. En este sentido, la intervención estatal se orienta al desarrollo de mecanismos para favorecer la competencia de mercado a través de un desmantelamiento de los sistemas que, históricamente, protegían a los trabajadores asalariados y de su sustitución por nuevas formas de flexibilidad. En segundo lugar, la reforma laboral establece como locus privilegiado de negociación la relación directa entre empleadores y trabajadores mediante la prevalencia de lo acordado en negociaciones colectivas o individuales por sobre aquello establecido en las leyes. 
En este contexto, la CUT ha desarrollado una estrategia de resistencia a la reforma, distanciándose de la posición negociadora y tendiente a la conciliación asumida durante los gobiernos petistas (Colombi, 2020). El giro en la estrategia de la principal central sindical de Brasil se vincula con las condiciones que presenta el nuevo escenario político e institucional: la central obrera pasó de ser una aliada al gobierno a ubicarse en un rol de oposición directa desde la llegada de Michel Temer, y se negó a aceptar y negociar ninguno de los puntos de la reforma laboral. Bajo estas condiciones, el eje de las acciones de la central se ubicó en el marco de una disputa electoral y defendió la candidatura de Lula como instrumento principal para frenar el retroceso de derechos (Colombi, Lemos y Krein, 2018). Tal estrategia tuvo un carácter limitado frente al impedimento de que Lula fuera candidato a la presidencia y el posterior triunfo de Jair Bolsonaro en las elecciones del año 2018.

En este cuadro general reviste importancia profundizar en la relación existente entre la reforma laboral del año 2017 y la estrategia llevada a cabo por el SM ABC, organización neurálgica de la CUT. Como veremos a continuación, al analizar el accionar del sindicato no como reacción frente a la reforma sino en su relación con ella, es posible identificar diferenciaciones, pero también trazos de continuidad respecto de la estrategia configurada durante los gobiernos del PT.

\subsection{Puntos de continuidad}

La descentralización de la negociación colectiva es uno de los aspectos que instituye la reforma laboral. No obstante, como se ha desarrollado previamente, en el caso del SM ABC esta tendencia se configura y consolida ya desde inicios de los años dos mil. Cabe señalar que hasta el año 2009 los sindicatos afiliados a la Federação Estadual dos Metalúrgicos (FEM-CUT/SP) llevaban a cabo negociaciones colectivas a nivel estatal mediante la firma de Convenciones Colectivas de Trabajo (CCT) con una vigencia de dos años. Esta dinámica se modifica a partir del año 2011, cuando comienzan a negociarse acuerdos colectivos firmados a nivel de empresa con una vigencia también de dos años. Al indagar sobre este cambio en la dinámica de negociación colectiva, un representante del CSE del SM ABC en la terminal automotriz Ford de São Bernardo do Campo indicaba lo siguiente:

A partir del año 2009, que fue el último acuerdo conjunto, algunas terminales automotrices comenzaron a manifestar que no conseguían pagar lo que pagaban otras. Toyota decía "Mirá, no consigo pagar lo que Ford paga”, General Motors decía "No consigo hacer lo que Volkswagen hace...". Y ahí comenzó a haber una necesidad de conversar de forma individualizada, pero siempre se sigue un parámetro. Entonces lo que una terminal automotriz da, el resto va a luchar para ir a buscar la misma cosa (Representante CSE - Comité Sindical de Ford São Bernardo do Campo, 22/07/2015).

La justificación discursiva y práctica de la negociación a nivel de empresa por sobre las negociaciones a nivel estatal por rama de actividad reside en el argumento de que cada empresa posee características distintas. Desde este punto de partida, se asume que el alcance de mejores condiciones de trabajo y salario está vinculado con el poder de negociación que los trabajadores puedan ejercer en el lugar de trabajo. Bajo esta lógica, en los años transcurridos entre 2003 y 2014, en la industria automotriz de la Región ABC de Brasil se fue consolidando un sistema de negociación colectiva en el cual las negociaciones al nivel de empresa se abrieron paso frente a las convenciones colectivas negociadas a nivel estatal. El SM ABC no sólo fue partícipe de este proceso sino que lo promovió.

En el año 2011, el SM ABC impulsó el Acordo Coletivo Especial (ACE), un proyecto de ley en el campo de la negociación colectiva que hoy se revela como una versión preliminar de algunos de los lineamientos sobre los cuales se desarrolló la reforma laboral en Brasil. El proyecto buscaba implementar un nuevo mecanismo de negociación colectiva a nivel de empresa sin mediación del Estado, ligado a las condiciones específicas de cada establecimiento y no a las relaciones laborales de un sector económico y/o categoría de trabajadores. En 
tal dirección, el ACE implicaba una profunda flexibilización de la legislación laboral, lo cual suscitó intensos debates al interior de la CUT y dificultó su presentación en el Parlamento.

Pese a que el ACE no fue implementado, resulta pertinente desarrollar algunos de sus fundamentos para comprender la orientación político-ideológica del SM ABC. Un documento elaborado por el sindicato expresa que el ACE parte de considerar que el poder otorgado al Estado en la regulación de las relaciones laborales de Brasil es excesivo:

La CLT [instrumento que regula las relaciones laborales en Brasil] acierta cuando fja parámetros básicos para regular una relación que siempre fue muy desigual entre capital y trabajo, posicionándose a favor del sector más débil. Confere al Poder Judicial instrumentos para asegurar un minimo de equilibrio en una anteposición que tradicionalmente es muy desequilibrada. Pero la ley erosiona la autonomía de los trabajadores, imponiendo una tutela del Estado, que, como toda tutela, se convierte en barrera para el establecimiento de un equilibrio más consistente. Donde existe control excesivo y reglas rigidas, la libertad muere (SM ABC, 2011, p. 13).

De este modo, el sindicato formuló una crítica y una propuesta de modificación a la legislación laboral de Brasil por no responder de forma adecuada a las necesidades de crecimiento económico del país. A través de la elaboración del ACE, el SM ABC cuestionó la importancia de flexibilizar los mecanismos de negociación colectiva estipulados en la CLT, amparándose en que la capacidad de organización y lucha alcanzada por los sindicatos en el lugar de trabajo sería la garantía para el alcance de mejores condiciones de trabajo. En este sentido, se asume que si bien la negociación a nivel empresa puede erosionar las protecciones de los segmentos profesionales menos organizados y movilizados, la clave no se encuentra en obstaculizar las posibilidades de negociación de los trabajadores con un mayor grado de organización sino en fortalecer la representación en el lugar de trabajo de aquellas categorías más débiles.

Con argumentos similares, la reforma laboral regula la representación de los trabajadores en el interior de aquellas empresas que posean más de doscientos trabajadores (tres a siete miembros, dependiendo del tamaño de la empresa). Este elemento es una novedad en la legislación laboral de Brasil en la cual, hasta el momento, la representación en el lugar de trabajo no estaba regulada por ley. Sin embargo, como señala Krein (2018), la nueva regulación no garantiza estabilidad laboral para los representantes electos. Esto significa que pueden ser despedidos en caso de crisis económica, introducción de nuevas tecnologías y problemas disciplinares. En concreto, se obstaculiza el desarrollo de una comisión de fábrica autónoma e independiente de la empresa y sus atribuciones se reducen a promover el diálogo y reducir los conflictos.

\subsection{Aspectos novedosos}

Ahora bien, no todo son líneas de continuidad. El cambio de signo político del gobierno significó para el SM ABC la oportunidad de desarrollar nuevos cursos de acción. En este sentido, una particularidad de los trabajadores metalúrgicos de Brasil ante la reforma laboral ha sido su actuación de forma unitaria en el movimiento Brasil Metalúrgico, iniciativa promovida por los principales sindicatos del sector ligados a distintas centrales sindicales (CONLUTAS, CUT, FS, Intersindical, CTB y UGT).

El objetivo de esta estrategia fue definir una unidad de acción para las campañas salariales de 2017 e impedir la reducción de derechos. La unidad de los trabajadores metalúrgicos se gestó en plenarios en los que se definieron iniciativas concretas con el objetivo de rechazar la reforma laboral. Bajo la consigna "Unirse y resistir: ningún derecho menos" se llevaron a cabo protestas y paralizaciones, particularmente en los días cercanos a la entrada en vigencia de la reforma laboral. Desde su conformación, el movimiento planteó el desarrollo de un plan de acción tendiente a promover la generación de empleos de calidad, defender la industria nacional, exigir el respeto al derecho de una jubilación digna, luchar contra la tercerización y defender las conquistas de las convenciones colectivas. Pese a ello, los sindicatos que forman parte del 
movimiento "Brasil metalúrgico" desarrollaron estrategias particulares al momento de negociar salario y/o condiciones de trabajo.

El SM ABC continuó llevando a cabo negociaciones por empresa y, en algunos casos, acciones de lucha como huelgas de brazos cruzados, paralizaciones y/o movilizaciones por lugar de trabajo en el marco de dichas negociaciones. Como señalan Colombi, Lemos y Krein (2018). tal estrategia ha sido efectiva. Hacia fines de octubre de 2017, el SM ABC anunció que a lo largo de ese año había firmado acuerdos colectivos con 62 empresas, que garantizaban la cobertura de derechos a más de 7.800 trabajadores. En tales acuerdos el sindicato pudo negociar por encima de la inflación, renovar las cláusulas sociales e introducir una cláusula de salvaguarda previendo que ningún cambio sería realizado a través de la reforma, sin negociación con participación del sindicato.

Sin embargo, la derrota el PT en las elecciones presidenciales del año 2018, el agravamiento de la crisis económica y el deterioro de la industria automotriz limitaron la estrategia del SM ABC. En este nuevo y adverso escenario, el SM ABC pudo resguardar el poder adquisitivo del salario negociando por encima de la inflación pero a cambio de una mayor flexibilización laboral a través de la aceptación de contenidos contemplados en la reforma laboral vinculados, principalmente, con la flexibilización de la jornada y las condiciones de contratación (Dieese/CUT, 2018).

\section{ReFLeXiones FinAles}

El día 7 de abril de 2018, en la histórica sede del Sindicato dos Metalúrgicos do $A B C$ ubicada en São Bernardo do Campo, Lula da Silva se dirigió a miles de militantes de la CUT y del PT que se habían movilizado para rechazar el mandato de prisión emitido por el juez Sergio Moro. Allí, el expresidente de Brasil desmintió las acusaciones, puntualizó cuáles habían sido las políticas sociales llevadas a cabo durante su gobierno y el de Dilma Rousseff, y también reivindicó el proceso de organización y resistencia configurado al calor de las huelgas de fines de los años setenta, cuando él presidía el SM ABC. En tal sentido, al referirse a la singularidad de aquel movimiento, Lula expresó lo siguiente:

El dato concreto es que nadie aguantó 41 dias [de huelga] porque, en la práctica, el compañero tenía que pagar la leche, tenia que pagar la cuenta de luz, tenía que pagar gas (...), entonces comenzó a sufrir presiones y no aguantó. Pero es "cómico" porque, en la derrota, ganamos mucho más sin ganar económicamente que cuando ganamos económicamente. Significa que no es el dinero lo que resuelve el problema de una huelga, no es $5 \%$, no es $10 \%$ : es lo que está incrustado de teoría politica, de conocimiento politico $y$ de tesis politica en una huelga (Brasil de Fato, 7/04/2018, traducción propia).

Estas palabras expresan el carácter que efectivamente tuvieron las huelgas impulsadas por los trabajadores metalúrgicos del $\mathrm{ABC}$ y la posterior constitución de la CUT y del PT: una central sindical y un partido político que funcionasen como herramientas de lucha para el mejoramiento de las condiciones materiales de los trabajadores y sus familias, pero, también, como espacios de organización de una clase frente a otra, de los trabajadores frente a sus empleadores. Así, en las palabras de Lula el aspecto político de las huelgas sindicales se ubica en primer plano al momento de delinear una estrategia de resistencia a la actual ofensiva.

Tras pasar 580 días en prisión, Lula fue liberado el 9 de noviembre de 2019. A las 48 horas de su liberación, y tras las repercusiones que habían tenido sus primeras declaraciones, el líder petista publicó en su cuenta de Twitter: "Yo no estoy más radicalizado. Estoy más consciente. Nunca leí tanto sobre la esclavitud como leí en la prisión. Y sé que ella no acabó” (Lula da Silva, cuenta personal de Twitter, 22/11/2019, traducción propia). Nuevamente, las contradicciones que signan las relaciones sociales capitalistas adquieren relevancia en el discurso del expresidente, orientado a fortalecer un espacio político de oposición capaz de enfrentar las políticas de austeridad del gobierno actual.

No concierne a los fines de este artículo indagar en qué medida las reminiscencias al novo sindicalismo describen el actual momento del PT y la CUT en la escena política brasileña. No obstante, a la luz de los 
procesos políticos suscitados en ese momento, el contenido de las palabras que recuperamos pone en primer plano una cuestión que en el último tiempo ha quedado relegada en los análisis sobre acción sindical: los límites de circunscribir la organización de la clase trabajadora al plano de la lucha económica. En efecto, a la luz de la experiencia brasileña es posible constatar que tal estrategia no ha sido fructífera y que es preciso recomponer y organizar los lazos sociales en un escenario signado por la pérdida de derechos; en particular, de aquellos asociados al trabajo.

En este marco, adquiere centralidad estudiar la relación entre reformas laborales y estrategias sindicales pero sin circunscribir el análisis a un abordaje dicotómico del tipo reforma laboral sí / reforma laboral no. En cambio, la propuesta del presente artículo estuvo orientada a considerar cuál es el rol que desempeñan los actores implicados en la configuración de los instrumentos normativos que regulan la relación capital-trabajo. En tal dirección, hemos analizado la relación entre estrategias sindicales y reformas laborales observando no sólo como reaccionan y/o se posicionan los sindicatos frente a los cambios en las formas de regulación sino también, y fundamentalmente, buscando conocer en qué medida el tipo de estrategia que desarrollan tiende a potenciar o, por el contrario, restringir las posibilidades de resistir a la implementación y posterior sanción de la reforma.

El caso del SM ABC pone de manifiesto las dificultades que presenta circunscribir el accionar sindical (y su análisis) al plano de la lucha económica, de la negociación de las condiciones de compra/venta y uso/ consumo productivo de la fuerza de trabajo y relegar aquella "otra función" de las organizaciones sindicales: la de organizar al conjunto de los trabajadores frente al capital. Recuperar la historia del SM ABC permite ver que concebir el accionar sindical de este modo no es una utopía y que la institucionalización de las luchas sindicales que comienza a observarse desde los años noventa y que se consolida en los años dos mil bajo los gobiernos del PT no responde únicamente al contexto económico, político e institucional, sino también a elementos que remiten a la estructura interna del sindicato, a los cambios en la orientación político ideológica de su accionar y a la relación que el movimiento sindical construye con el gobierno.

A la luz del escenario que comienza a delinearse en América Latina, este texto busca destacar la utilidad e incidencia que en el campo sindical y político adquiere el modo en que se formulan los análisis provenientes del ámbito académico. En este sentido, el estudio de las estrategias sindicales circunscripto a las luchas económicas, reivindicativas y situadas en un plano institucional, reproduce limitaciones a la hora de reflexionar acerca de los procesos de reorganización que atraviesan los sindicatos y, lo que es aún más importante, nubla aquel horizonte de posibilidades que tiene el accionar sindical.

\section{FUENTES DOCUMENTALES UTILIZADAS}

\section{Documentos sindicales}

Sindicato dos Metalúrgicos do ABC (SM ABC) (2011). ACE, Acordo Coletivo Especial. San Pablo: Sindicato dos Metalúrgicos do ABC.

Tribuna Metalúrgica No 1889 (14 de septiembre de 2004). Recuperado de https://smabc.org.br/tribuna/e dicao-no-1889/

Tribuna Metalúrgica No 1997 (13 de mayo de 2005). Recuperado de https://smabc.org.br/tribuna/edicao -no-1997/

Tribuna Metalúrgica No 2000 (19 de mayo de 2005). Recuperado de https://smabc.org.br/tribuna/edicao -no-2000/

Tribuna Metalúrgica No 2008 (7 de junio de 2005). Recuperado de https://smabc.org.br/tribuna/edicaono-2008/ 


\section{Multimedia}

Da Silva, L. I. (2019). Twitter. Recuperado de https://twitter.com/LulaOficial/status/119806821693486 2848.

\section{Agradecimientos}

Agradezco los valiosos comentarios de los/as evaluadores/as anónimos/as de la Revista Sociohistórica, cuyas observaciones han sido muy importantes para enriquecer los argumentos planteados en el artículo.

\section{REFERENCIAS}

Antunes, R. (1988). A rebeldia do trabalho. Campinas: UNICAMP

Atzeni, M., y Ghigliani, P. (2008). Nature and limits of trade unions' mobilisations in contemporary Argentina. Labour Again Publications. Recuperado de http://www.iisg.nl/labouragain/labourargentina.php

Asociación de Abogados Laboralistas (2018). Reforma laboral. Documento critico sobre los proyectos de ley presentados por el Poder Ejecutivo Nacional. Recuperado de https://drive.google.com/file/d/1D58s0Qrc5VO_n_9V7emm cogiQT1bq6jU/view

Bensusán, G. (2017). Nuevas tendencias en el empleo: retos y opciones para las regulaciones y politicas del mercado de trabajo. Recuperado de CEPAL https://www.cepal.org/sites/default/files/document/files/bensusan_1.1.pdf

Boito, A. (1994). De volta para o corporativismo: a trajetória política do sindicalismo brasileiro. São Paulo Em Perspectiva, 8(3) 23-28. Recuperado de http://produtos.seade.gov.br/produtos/spp/v08n03/v08n03_04.pdf

Boito, A. y Marcelino, P. (2010). O sindicalismo deixou a crise para trás? Um novo ciclo de greves na década de 2000. Caderno CRH, 23(59), 323-338. http://dx.doi.org/10.1590/S0103-49792010000200008

Boito Jr., A., Galvão, A. y Marcelino, P. (2011). Brasil: o movimento sindical e popular na década de 2000. En M. Modonessi y J. Rebón (Compiladores), Una década en movimiento. Luchas populares en América Latina en el amanecer del siglo XXI (pp.153-182). Buenos Aires: CLACSO. Prometeo. UBA Sociales Publicaciones. Facultad de Filosofía y Letras /UBA.

Colombi, A. P. (2020). Trabalho e ação coletiva nos governos do pt: a atuação da cut e da fs entre os anos 2003 e 2014. Belo Horizonte: Fino traço editora

Colombi, A. P., Lemos, P. R. y Krein, J. D. (2018). Entre negociação e mobilização: as estratégias da CUT e da FS frente à reforma trabalhista no Brasil. Revista Brasileira de Estudos do Trabalho (ABET).17(2), 179-198. https ://doi.org/10.22478/ufpb.1676-4439.2018v17n2.44618

Colombi, A. P. y D’Urso, L. (2018). Ação sindical na Argentina e No Brasil: uma análise a partir dos casos da CUT e da CGT no alvorecer do século XXI. Revista da Associação Brasileira de Estudos (ABET). 17(1), 154-175. htt ps://doi.org/10.22478/ufpb.1676-4439.2018v17n1.41173

Connolly, H. y Darlington, R. (2012). Radical political unionism in France and Britain: A comparative study of SUDRail and the RMT. European Journal of Industrial Relations, 18(3), 235-250. https://doi.org/10.1177/09596 80112452693

DIEESE/CUT (2018). Acompanhamento das negociaçôes coletivas após reforma trabalhista. San Pablo: DIEESE/CUT

De Souza, C. D. y Vieira Trópia, P. (2012). O protagonismo metalúrgico no sindicalismo brasileiro. En C. D. De Souza y P. Vieira Trópia (Eds.), Sindicatos Metalúrgicos No Brasil Contemporâneo (pp. 13-46). Belo Horizonte: Fino Traço.

Del Bono, A. (2018). Economía de Plataforma. Nuevo modelo de explotación laboral. Revista Mestiza. Recuperado de https://revistamestiza.unaj.edu.ar/nuevo-modelo-de-explotacion-laboral/

Departamento Intersindical de Estatística e Estudos Socioeconômicos - DIEESE. (2005). O movimiento grevista em 2004. Recuperado de https://www.dieese.org.br/balancodasgreves/2004/estpesq12102005_greve2004.pdf 
Departamento Intersindical de Estatística e EstudosSocioeconômicos - DIEESE. (2013). Balanço das greves em 2012. Recuperado de https://www.dieese.org.br/balancodasgreves/2012/estPesq66balancogreves2012.pdf

Fishwick, A. y Connolly, H. (2018). Austerity and Working-Class Resistance. Survival, Disruption and Creation in Hard Times. Londres: Rowman \& Littlefield International

Galvão, A. (2012). De "laboratório" das relações de trabalho a formulador da politica nacional: o Sindicato dos Metalúrgicos do ABC paulista. En D. C. De Souza y P. Trópia (Eds.), Sindicatos Metalúrgicos No Brasil Contemporâneo (pp. 135-162). Belo Horizonte: Fino Traço

Galvão, A. (2002). A CUT na encrusilhada: dilemas do movimiento sindical combativo. Ideias, 9(1), 105-54

Galvão, A. (1998). Os metalùrgicos do ABC e a cámara setorial da indústria automovilística. Revista de Sociologia e Politica, (10/11), 83-101. Recuperado de https://revistas.ufpr.br/rsp/article/view/39278/24097

Hyman, R. (2001). Understanding European trade unionism: between market, class and society. Londres: Sage

Krein, J. D. (2018). O desmonte dos direitos, as novas configurações do trabalho e o esvaziamento da ação coletiva: consequências da reforma trabalhista. Tempo social, 30(1), 77-104. http://dx.doi.org/10.11606/0103-2070.ts. 2018.138082

Krein, J. D. (2013). As Relações de Trabalho na Era do Neoliberalismo no Brasil. Campinas-SP: Editora LTr, IE/ UNICAMP.

Krein, J. D. y Gimenez, D. M. (2018). Dimensões críticas da reforma trabalhista no Brasil. Apresentação. Campinas: Editora Curt Nimuendajú.

Krein, J. D. y Teixeira, M. O. (2014). As controvérsias das negociações coletivas nos anos 2000 no Brasil. En R. Verás de Oliveira, M. A. Bridi y M. Ferras (Eds.), O Sindicalismo na Era Lula: paradoxos, perspectivas e olhares (pp. 213-246). Belo Horizonte: Fino Traço.

Futema, F. (18 de mayo de 2005). Greve paralisa Volksem São Bernardo e GM em Mogi das Cruzes. Folha Online. Recuperado de http://www1.folha.uol.com.br/folha/dinheiro/ult91u96397.shtml

Ladosky, G., Ramalho J. R. y Rodrigues, I. J. (2014). A questão trabalhista e os desafios da ação sindical nos anos 2000. En R. Verás de Oliveira, M. A. Bridi y M. Ferras (Eds.), O Sindicalismo na Era Lula: paradoxos, perspectivas e olhares (pp. 61-86). Belo Horizonte: Fino Traço.

Marticorena, C. (2018). La reforma laboral que quiere Cambiemos. Revista Punto Crítico. Recuperado de https://re vistapuntocritico.wordpress.com/2018/04/16/318/

Noronha, E. G. (2009). Ciclo de greves, transição política e estabilização: Brasil, 1978-2007. Lua Nova, (76), 119168. http://dx.doi.org/10.1590/S0102-64452009000100005

Praun, L. (2012). Sindicalismo metalúrgico no ABC paulista: da contestação a parceria. En D. Cangassu de Sousa y P. Vieira Trópia (Eds.), Sindicatos Metalúrgicos No Brasil Contemporâneo (pp. 109-134.) Belo Horizonte: Fino traço

Redacción (7 de abril de 2018). Leia a íntegra do discurso histórico de Lula em São Bernardo. Brasil de Fato. Recuperado de https://www.brasildefato.com.br/2018/04/07/leia-a-integra-do-discurso-historico-de-lula-emsao-bernardo/

Rolli, C., Amato, F. y Do Valle, D. (11 de septiembre de 2009). Trabalhadores ampliam paralisações nas montadoras por aumento salarial. Folha Online. Recuperado de http://www1.folha.uol.com.br/folha/dinheiro/ult91u6224 60.shtml

Sader, E. (1988). Quando novos personagens entramem cena: experiências, falas e luta dos trabalhadores da Grande São Paulo (1970-80). San Pablo: Paz e terra.

Trópia, P. V. (2009). Força Sindical: politica e ideologia no sindicalismo brasileiro. San Pablo: Expressão Popular.

Upchurch, M. y Mathers, A. (2012). Neoliberal Globalization and Trade Unionism: Toward Radical Political Unionism? Critical Sociology, 38(2), 265-280. https://doi.org/10.1177/0896920510396384

Véras de Oliveira, R. (2011). Sindicalismo e democracia no Brasil: do novo sindicalismo ao sindicato cidadão. San Pablo: Annablume. 
Sociohistórica, $\mathrm{n}^{\circ}$ 48, e143, septiembre 2021-febrero 2022. ISSN 1852-1606

\section{Notas}

1 Tribuna Metalúrgica No 1997 (13 de mayo de 2005).

2 Tribuna Metalúrgica No 2008 (7 de junio de 2005). 